\title{
Application of the Kano-Hamilton Multiangle Inversion Method in Clear Atmospheres
}

\author{
MARIANA ADAM \\ Department of Geography and Environmental Engineering, The Johns Hopkins University, Baltimore, Maryland \\ Vladimir A. Kovalev, Cyle Wold, and Jenny Newton \\ Fire Science Laboratory, U.S. Department of Agriculture, Missoula, Montana \\ MARKus PAHLOW \\ Institute of Hydrology, Water Resources Management and Environmental Engineering, Ruhr-University Bochum, Bochum, Germany
}

WeI M. HaO

Fire Science Laboratory, U.S. Department of Agriculture, Missoula, Montana

Marc B. Parlange

School of Architecture, Civil and Environmental Engineering, École Polytechnique Fédérale de Lausanne, Lausanne, Switzerland, and Department of Geography and Environmental Engineering, The Johns Hopkins University, Baltimore, Maryland

(Manuscript received 30 November 2006, in final form 12 March 2007)

\begin{abstract}
An improved measurement methodology and a data-processing technique for multiangle data obtained with an elastic scanning lidar in clear atmospheres are introduced. Azimuthal and slope scans are combined to reduce the atmospheric heterogeneity. Vertical profiles of optical depth and intercept (proportional to the logarithm of the backscatter coefficient) are determined. The purpose of this approach is to identify and remove data points that distort the regression analysis results in order to improve the accuracy of the retrieved optical depth and of the intercept. In addition, the influence of systematic distortions has been investigated. Furthermore, profiles of the optical depth, intercept, and the range-squared-corrected signals have been used to determine the lidar overlap function as a function of range. Simulation and experimental results of this data-processing technique are presented.
\end{abstract}

\section{Introduction}

The method proposed by Kano (1968) and Hamilton (1969) for the inversion of data obtained with a scanning lidar is currently the only method available to obtain extinction profiles from elastic backscatter data without making assumptions about the backscatter-toextinction ratio. The Kano-Hamilton method assumes that the atmosphere is horizontally stratified, implying that backscatter and extinction coefficients are invari-

\footnotetext{
Corresponding author address: Mariana Adam, Dept. of Geography and Environmental Engineering, The Johns Hopkins University, Baltimore, MD 21218.

E-mail: mariana@jhu.edu
}

DOI: $10.1175 / 2007 J T E C H A 946.1$

(C) 2007 American Meteorological Society ant in horizontal layers. The real atmosphere is, of course, not homogeneous, but in most cases the horizontal variability is significantly less than vertical changes in aerosol loading, at least for local ranges under consideration. Therefore, the assumption of horizontal homogeneity is often more reasonable than the assumption of a height-independent backscatter-toextinction ratio (or lidar ratio), which is used in most one-directional, vertical elastic-lidar measurements. In fact, the inversion results obtained with onedirectional measurements based on an invariant lidar ratio are known to be inaccurate [see, e.g., the study done by the European Aerosol Research Lidar Network (EARLINET) community (Böckmann et al. 2004)].

Unfortunately, multiangle methods, which do not re- 
quire a height-independent backscatter-to-extinction ratio, can also yield poor inversion results (Spinhirne et al. 1980; Russell and Livingston 1984; Rothermel and Jones 1985) and are rarely used in lidar measurements (Spinhirne et al. 1980; Russell and Livingston 1984; Rothermel and Jones 1985; Sicard et al. 2002; Takamura et al. 1994; Sasano 1996; Kovalev and Eichinger 2004; Pahlow et al. 2004; Kovalev et al. 2004). Atmospheric heterogeneity is commonly pinpointed as the major reason for poor accuracy of these methods. We show in this paper that the problem of multiangle measurements goes beyond atmospheric heterogeneity. To obtain a comprehensive understanding of the specifics of multiangle measurements, we perform numerical simulations, followed by analyses of experimental data obtained at the Fire Science Laboratory (FSL) test site located in mountainous terrain, approximately $30 \mathrm{~km}$ west of the city of Missoula, Montana, at an altitude of $\sim 1000 \mathrm{~m}$. The measurements were made in clear atmospheres.

The paper is structured in three parts: an improved measurement methodology and data-processing technique for the Kano-Hamilton method is introduced first, followed by the determination of the lidar overlap function, and closing with a summary. The primary goal of this study is to test a measurement methodology and a data-processing technique that may improve the accuracy of the retrieval results when using the KanoHamilton multiangle method. The second goal is to accurately determine the lidar overlap function. To do this, it is critical to identify the optimal height interval $\left(h_{\min }, h_{\max }\right)$ that needs to be used to obtain accurate inversion results. To achieve this, the effects of systematic distortions, in addition to the commonly considered signal noise, need to be taken into account.

\section{Measurement methodology and data-processing techniques for the Kano-Hamilton method}

A short outline of the Kano-Hamilton multiangle method is provided below. It is followed by numerical simulations to investigate the influence of systematic distortions in lidar signals (in particular caused by a remaining signal offset) on inversion results. These results allow us to establish criteria that are used to determine the optimal height interval $\left(h_{\min }, h_{\max }\right)$ that is applied for inversion. In the second part we present results obtained from the analysis of experimental data.

\section{a. Theory and numerical simulations}

With the assumption of a horizontally stratified atmosphere, the lidar signals $P_{j}(h)$, measured along the elevation angle $\varphi_{j}$, can be written as (Kano 1968; Hamilton 1969)

$$
P_{j}(h)=C q_{j}(h) \beta(h)\left(h / \sin \varphi_{j}\right)^{-2} \exp \left[\frac{-2 \tau(0, h)}{\sin \varphi_{j}}\right],
$$

where $C$ is a lidar system constant and $q_{j}(h)$ is the overlap function at height $h$ for each elevation angle $\varphi_{j} ; \beta(h)$ is the total (molecular and particulate) backscatter coefficient, that is, $\beta(h)=\beta_{m}(h)+\beta_{p}(h)$; and $\tau(0, h)$ is the total optical depth from ground level to height $h$, including molecular and particulate components, $\tau(0$, $h)=\tau_{m}(0, h)+\tau_{p}(0, h)$. The dependence of the KanoHamilton function $y_{j}(h)$, defined as (Kovalev and Eichinger 2004)

$$
y_{j}(h)=\ln \left[P_{j}(h)\left(h / \sin \varphi_{j}\right)^{2}\right],
$$

taken versus independent variable $x_{j}=\left(\sin \varphi_{j}\right)^{-1}$, can be written as

$$
y_{j}(h)=A(h)-2 \tau(0, h) x_{j},
$$

where $P_{j}(h)\left(h / \sin \varphi_{j}\right)^{2}$ is the range-squared-corrected lidar signal measured at the height $h$ along the elevation angle $\varphi_{j}$, and

$$
A(h)=\ln \left[C q_{j}(h) \beta(h)\right] .
$$

The least squares analysis is applied using lidar data measured along different elevation angles to obtain the vertical optical depth $\tau(0, h)$ [Eq. (3)]. However, the constants $A(h)$ and $\tau(0, h)$ in Eq. (3) may be found only if the overlap function at $h$ in Eq. (4) does not depend on $\varphi_{j}$. To achieve this, only those lidar signals within the zone of complete overlap, where $q_{j}(h)=1$, should be used. Then Eqs. (3) and (4) become

$$
y_{j}(h)=A^{*}(h)-2 \tau(0, h) x_{j}
$$

and

$$
A^{*}(h)=\ln [C \beta(h)] .
$$

Accordingly, the minimal height for each slope direction at which the lidar data can be used for the inversion is defined by the length of the lidar incompleteoverlap zone.

Multiangle measurements are influenced by at least three major sources of uncertainty. The first source, widely discussed in the literature, is related to the determination of the slope in lidar data. Similar to differential absorption lidar (DIAL), high spectral resolution lidar (HSRL), and Raman lidar measurements (Whiteman 1999), the quantity that is regressed in the multiangle measurements $\left[\ln P_{j}(h)\left(h / \sin \varphi_{j}\right)^{2}\right]$ is in general not normally distributed. Various methods have been proposed to improve the accuracy of the slope determination in lidar data (Kunz and Leeuw 1993; 
Rocadenbosch et al. 1998, 2000, 2004; Volkov et al. 2002; Whiteman 1999; Kovalev 2002).

Here we focus on the other two sources of measurement uncertainty. The first is the systematic distortion due to the signal offset that remains after subtraction of the signal background component. The offset may be related to low-frequency noise components (e.g., electric interferences), a restricted frequency range of the photoreceiver, and/or receiver optics aberrations. Another source of uncertainty stems from using an imperfect measurement methodology and the multiangle inversion technique itself (e.g., a nonappropriate height interval used for inversion).

In our numerical simulations we consider the case of a lidar operating at $355 \mathrm{~nm}$ with signals corrupted by random noise in a layerwise horizontally homogeneous, clear atmosphere. The atmospheric particulate extinction coefficient at this wavelength is assumed to decrease exponentially from $0.1 \mathrm{~km}^{-1}$ at ground level to $0.001 \mathrm{~km}^{-1}$ at a height of $h=4600 \mathrm{~m}$. The incompleteoverlap zone extends up to $h=1000 \mathrm{~m}$, and the lidar operates along discrete elevation angles of $6^{\circ}, 7.5^{\circ}, 9^{\circ}$, $12^{\circ}, 15^{\circ}, 18^{\circ}, 22^{\circ}, 26^{\circ}, 32^{\circ}, 40^{\circ}, 49^{\circ}, 58^{\circ}, 68^{\circ}$, and $80^{\circ}$. With an assumed standard vertical profile of the molecular extinction coefficient, the vertical molecular optical depth, $\tau_{\text {mol }}(0, h)$, and the particulate component, $\tau_{\text {part }}(0, h)=\tau(0, h)-\tau_{\text {mol }}(0, h)$, can then be calculated. The maximal lidar range, $r_{\max , j}$, at which the lidar data are still acceptable for inversion, needs to be established. Here we chose $r_{\text {max }, j}$ as the location where the signal-to-noise ratio (SNR) is unity. Thus, all the signals with SNR $>1$ are used for inversion in the numerical simulations.

Taking into account that the length of the incomplete overlap has to be determined accurately, we first investigate the influence of data points that lie within the zone of incomplete overlap on the inversion results. In the upper plot of Fig. 1, the particulate optical depth as a function of height, obtained with multiangle inversion using simulated data, is shown. The signal noise level has a standard deviation (STD) of 1 count (arbitrary units), while the maximum signals reach $3100-3300$ counts. In the middle plot of Fig. 1 the intercept, $A^{*}(h)$, is shown as a function of height. The model overlap function used for the simulations is shown in the bottom part of Fig. 1. To demonstrate potential inversion problems that can arise when an incorrectly defined incomplete-overlap range is used (i.e., using points outside the complete-overlap zone), we erroneously assume here that the complete overlap starts at $500 \mathrm{~m}$, whereas the correct range is $1000 \mathrm{~m}$. Because of this error, points that belong to the zone of incomplete overlap are included in the inversion, resulting in the
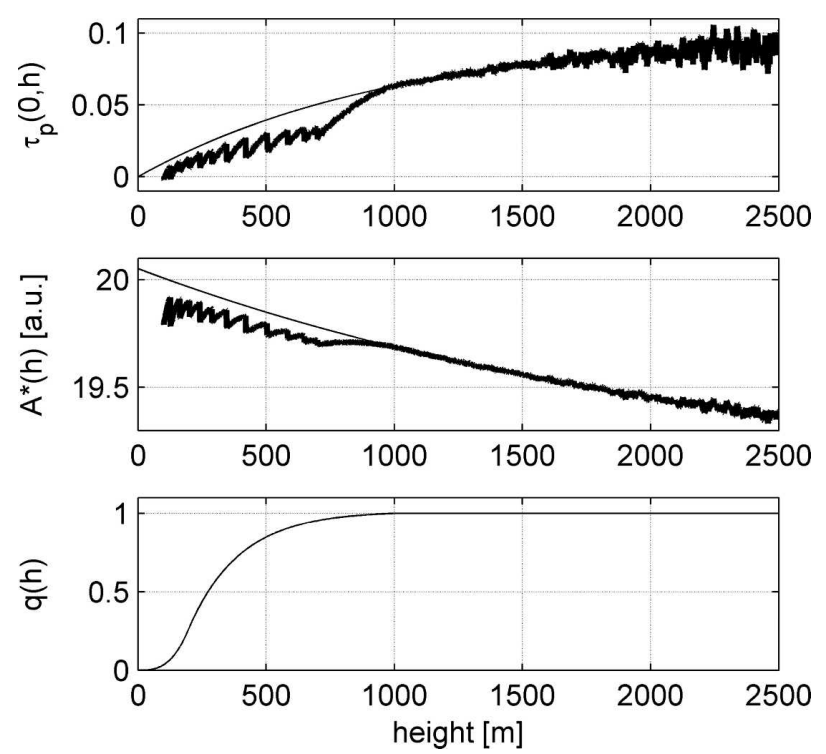

FIG. 1. Model dependence of the (top) particulate optical depth and (middle) intercept $A^{*}(h)$ with height (thin curves), and those obtained from the multiangle method with a virtual lidar (thick curve) under the condition of erroneous estimation of the length of the incomplete-overlap zone (500 m instead of $1000 \mathrm{~m}$ ). (bottom) The overlap function. The signals are corrupted with noise with an STD of 1 count.

divergence between the model and retrieved profiles up to a range of $\sim 1000 \mathrm{~m}$. Note that the optical depth and the intercept are underestimated in the near field. The optical depth also reaches a zero value at a height of around $100 \mathrm{~m}$ [this effect was also observed, but not explained, in the study by Sicard et al. (2002)]. This illustrates that it is crucial to determine the length of the incomplete overlap correctly. On the other hand, an incorrectly selected incomplete-overlap zone can be revealed by analysis of the nearest zone of the retrieved optical depth (see Fig. 1).

We now address the problem that arises from underestimating or overestimating the background component of the lidar signals. Either error in the estimated background component introduces systematic shifts (or offsets) in the background subtracted lidar signal. As a result, the measured backscatter signal is the sum of the real backscatter signal and the remaining offset, that is, $P_{\text {measured }}=P_{\text {real }}+P_{\text {offset }}$. These shifts can distort the derived profiles substantially at distant measurement ranges. To illustrate this, Fig. 2 shows the retrieved particulate optical depth $\tau_{p}(0, h)$ and the intercept $A^{*}(h)$, obtained from signals containing nonzero offsets [the upper curve represents an overestimated background with 1 count (i.e., offset $=-1$ ), while the lower curve represents an underestimated background with 1 count (i.e., offset $=+1$ count)]. Note that in this case 

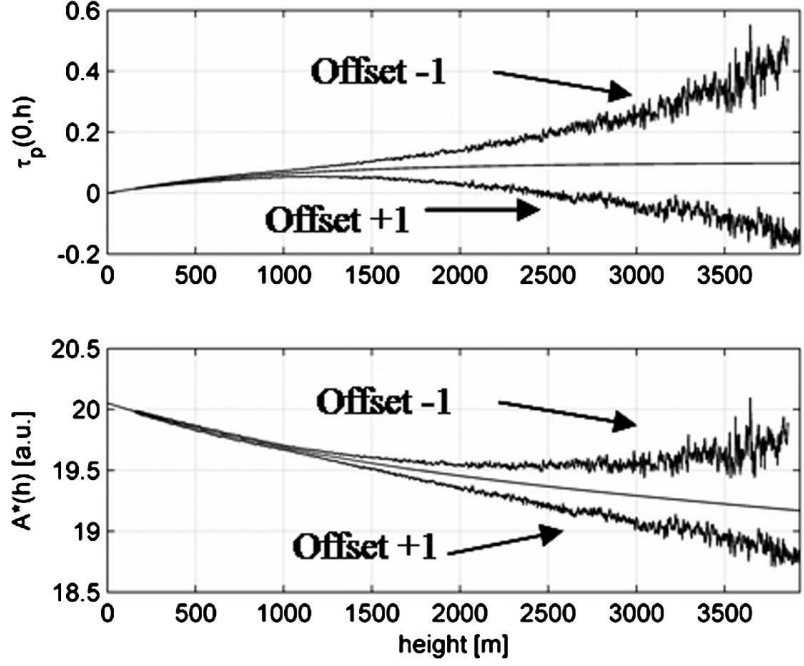

FIG. 2. (top) The model and retrieved particulate optical depth profiles obtained from the simulated multiangle measurements for a remaining offset in the signals (the thin and bold curves, respectively). The lower and upper bold curves show the retrieved particulate optical depth profiles where an offset of \pm 1 count remains in the signals after the background subtraction. (bottom) The retrieved intercept. The thin curve represents the model, while the lower and the upper bold curves represent the retrievals for the cases of an offset with \pm 1 count. The signals are corrupted with noise with an STD of 1 count.

the zone of complete overlap has been chosen correctly. The same model for the particulate optical depth dependence as that in Fig. 1 is used. The length of the incomplete overlap $(1000 \mathrm{~m})$ is chosen correctly. As before, the signal random noise has an STD of 1 count. The maximum signals are 3100-3300 counts. Note the strong deviation of the retrieved profile from the model profile over distant ranges.

To reduce measurement uncertainty arising from the aforementioned sources we apply the following procedure for measurements and data processing:

Lidar measurements at preselected elevation angles are taken. To reduce the influence of local atmospheric horizontal heterogeneity, at each elevation angle a sequence of 50 azimuthal scans with $1^{\circ}$ resolution is obtained, and a mean signal is calculated and used for the inversion. We start with the smallest elevation angle, take 50 azimuthal scans, move to the next elevation angle, take another 50 azimuthal scans, and so on, until we complete this set of scans at the last (largest) elevation angle. The mean signal is computed as follows. First, a mean and the standard deviation over the last 200 range bins (i.e., in this particular case $1200 \mathrm{~m}$ ) of the 50 measured signals are computed for each elevation angle. Profiles outside the boundaries defined by the mean \pm 1 STD are excluded from the dataset over the total measurement range. The signal mean and STD are recalculated with this reduced dataset for the entire range (see Fig. 3). This STD is taken as a measure of the errors in the data due to signal noise and to possible heterogeneities in the atmosphere and is accounted for in the propagation of the errors in the process of the calculations (see the appendix). For each direction, characterized by the elevation and azimuth angles, 30 shots were averaged. The background is subtracted from the mean signal, and SNR is defined as the ratio between the background subtracted mean signal and its STD (see the appendix). The range of the signals used in further analyses is constrained by the additional condition of SNR $\geq 5$ (Utkin et al. 2002).

The conventional Kano-Hamilton multiangle procedure is then applied, and the function $y_{j}(h)$, as defined in Eq. (2), is calculated and plotted versus $x_{j}$. After exclusion of all data points that do not satisfy the predefined conditions [i.e., outliers, in the function $y_{j}(h)$, as discussed below], the linear regression for the function $y_{j}(h)$ versus $x_{j}$, applied for each height $h$, is computed, and the regression constants, the intercept $A^{*}(h)$, and the total optical depth $\tau(0, h)$ are calculated (see Fig. 6$)$.

Note that the spatial (horizontal) averaging is often more desirable than the temporal averaging over a single direction. The analysis of the recorded horizontal scans allows us to exclude heterogeneities (local cloud or cloud tatter that is present only over a local area) by means of the statistical analysis. This is not possible with a one-directional scanning, where sometimes the only option is to stop the measurements until the cloud passes by (if wind blows in the right direction) or disappears. The main objective of this procedure is to determine the optimal height interval $\left(h_{\min }, h_{\max }\right)$ for which best inversion results are obtained. As shown within the simulations section, an important consideration in lidar data processing is the subtraction of the signal background component. Even minor uncertainty in the estimated background component can substantially affect the measurement accuracy over distant ranges (see Fig. 2 and also Kovalev 2004). The aggravating problem is that the actual background component level can be masked by low-frequency noise, so that the signal decreases slightly but continuously over the entire far-end range. This problem has been widely discussed in the literature (see, e.g., Lee et al. 1990; Zhao 1999; Bristow 2002; Fang and Huang 2004).

The calculations of $A^{*}(h)$ and $\tau(0, h)$ are performed 

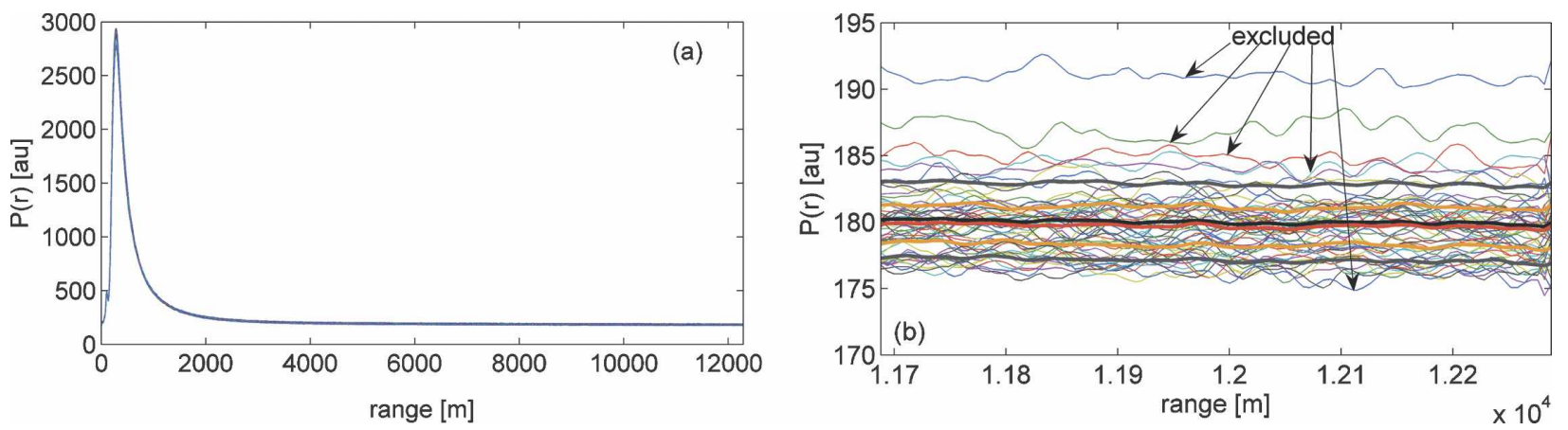

FIG. 3. Horizontal scan taken at elevation angle of $6^{\circ}$ : (a) $P(r)$ for all 50 azimuthal angles (which are undistinguishable) and (b) zoom in of (a) over the last $600 \mathrm{~m}$ of the total range. The thick black curve represents the mean, and the gray thick curves represent the STD over all 50 azimuthal angles. Profiles lying outside the boundaries defined by the STD (outside the gray curves) are excluded from the dataset (see arrows). The thick red curve represents the final mean, while the thick orange curves represent the STD obtained after exclusion of those signals.

over an optimal height interval $\left(h_{\min }, h_{\max }\right)$. The criteria for determining an optimal height interval are as follows.

1) The individual maximal range, $r_{\text {max }, j}$, is given by the condition SNR $>5$ [or $P(r) \geq 5 \sigma_{P(r)}$ ]. The corresponding maximal height is given by $h_{\text {max }, j}=r_{\text {max }, j}$ $\sin \varphi_{j}$ for each profile.

2) It is well known that points from incomplete overlap should not be used for the inversion. Therefore, data points on the left-hand side of the maximum value of $y_{j}(h)$ are not used for the regression. Moreover, to reduce the influence of possible systematic distortions of the recorded backscatter signal in the range of its sharp reduction, $h_{\min }$ was slightly shifted to the right-hand side of the maximal $y_{j}(h)$.

3) At the maximal height, $h_{\max }$, a minimal number, $n_{\text {min }}$, of points is needed for the regression. For example, in the case shown here, out of the 14 elevation angles we used $n_{\min }=6$ points. Elsewhere $(h<$ $\left.h_{\text {max }}\right)$, the minimum number of points for regression was restricted to 3 . With these criteria, the optimal heights interval for $y_{j}$ is established, and the func-

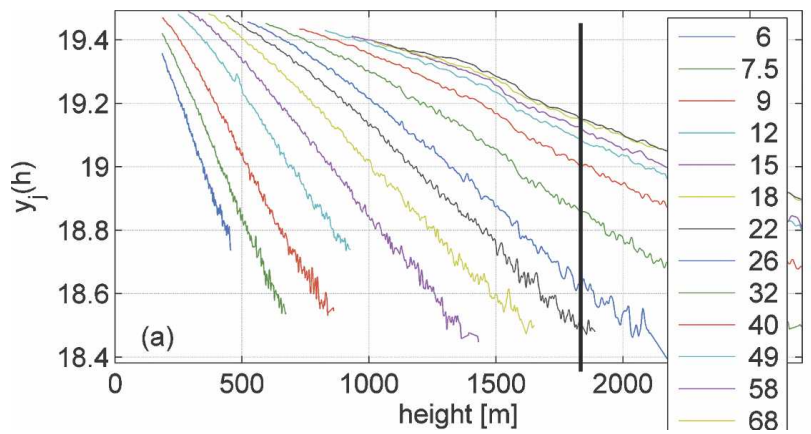

tions $\tau(0, h)$ and $A *(h)$ can be calculated from Eq. (5) using a weighted linear regression (see the appendix) as a function of $\sigma_{P}$ (see, e.g., Barlow 1989; Taylor 1997; Whiteman 1999). This is illustrated with an example shown in Fig. 4. Applying criteria 1-3 leads to $h_{\min }=h_{\min , 3}$, while $h_{\max }=h_{\max , 9}$. Note that criteria 1 and 2 provide the individual $h_{\min , j}$ and $h_{\text {max }, j}$ (and the corresponding $r_{\min , j}$ and $r_{\max , j}$ ), while criterion 3 defines the optimal height interval $\left(h_{\min }\right.$, $h_{\max }$ ) (Fig. 5).

A qualitative analysis of both functions, $\tau(0, h)$ and $A^{*}(h)$, helps to draw preliminary conclusions about the quality of the lidar data and, particularly, on whether the atmospheric conditions are favorable for applying the multiangle inversion algorithms. There are at least two simple conditions for $\tau_{p}(0, h)$ that allow for an initial estimate of the quality of the retrieved lidar data: (i) the particulate optical depth, $\tau_{p}(0, h)$, has to be positive and must be monotonically increasing and (ii) the function $\tau_{p}(0, h)$ should tend to zero when extrapolating $\tau_{p}(0, h)$ to $h=0$. Note that most of the experimental data obtained at $1064 \mathrm{~nm}$ had to be rejected because

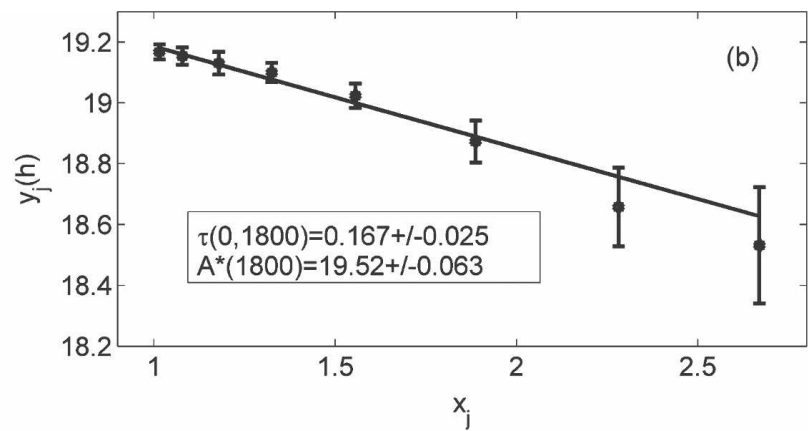

FIG. 4. Example of linear regression to determine $\tau(0, h)$ and $A^{*}(h)$ for a particular height. (a) In this example, the regression is determined for $h=1800 \mathrm{~m}$. (b) Values of $y_{j}\left(h=1800 \mathrm{~m}\right.$ ) are plotted with respect to $x_{j}$. 


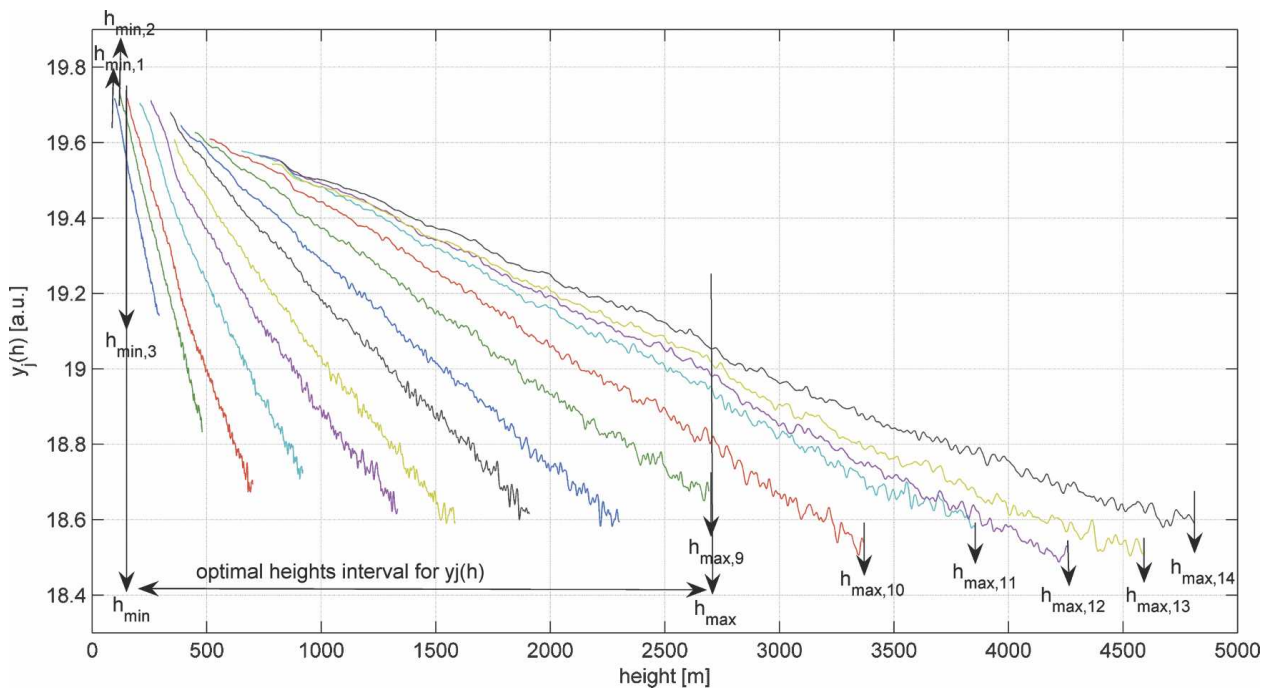

FIG. 5. Example of selecting the optimal heights interval for $y_{j}(h)$ used for inversion. Criterion 1 gives us $h_{\max , j}$, while criterion 2 gives us $h_{\min , j}$. Criterion 3 selects the optimal height interval such that $h_{\min }=$ $h_{\min , 3}$ and $h_{\max }=h_{\max , 9}$.

they did not satisfy condition (i) and sometimes also (ii) and, therefore, it was considered suitable to stop performing further inversion of these data.

\section{b. Experiment}

The experiment was performed on six days between 28 February and 6 April 2005, and several sets (sequences) of data were obtained per day with two lidars [FSL and The Johns Hopkins University (JHU)]. The experiments took place under clear atmospheric conditions. During the experiment, the electronic noise in the FSL lidar data was generally much less than in the JHU lidar data; therefore, the results of the former are mainly discussed. A few examples are shown to evaluate the agreement between the two lidars. The techni- cal characteristics of the FSL and JHU lidars are given in Table 1. The digitizers have a 56-MHz low-pass filter. We used a spatial resolution of $6 \mathrm{~m}$, corresponding to a $25 \mathrm{MS} \mathrm{s}^{-1}$ digitizer rate. Measurements were carried out using 14 elevation angles, the same that were used for the numerical simulations. This provided a sufficiently large number of points for regression. The total time required for a full azimuthal and vertical scan was approximately $30 \mathrm{~min}$. The elevation angle's pointing resolution of the lidar is $\sim 0.0018^{\circ}$, the pointing ambiguity due to gear lash is up to $0.34^{\circ}$, and the leveling error is less than $0.5^{\circ}$.

In clear atmospheres, where the particulate loading is comparable with the molecular loading, the basic assumption of the Kano-Hamilton method (horizontally

TABLE 1. Lidar system parameters.

\begin{tabular}{|c|c|c|}
\hline & FSL lidar & JHU lidar \\
\hline Laser & Q-switch Nd:YAG & Q-switch Nd:YAG \\
\hline Wavelengths & $1064,355 \mathrm{~nm}$ & $1064,532,355 \mathrm{~nm}$ \\
\hline Receiver & 25.4-cm Cassegrain telescope & 25.4-cm Cassegrain telescope \\
\hline Detectors & $\begin{array}{l}1064 \mathrm{~nm} \text { : chilled, IR-enhanced Si Avalanche } \\
\text { Photodiode } \\
355 \mathrm{~nm} \text { : photomultiplier }\end{array}$ & $\begin{array}{l}1064 \mathrm{~nm} \text { : IR-enhanced Si Avalanche Photodiode } \\
355 \text { and } 532 \mathrm{~nm} \text { : photomultiplier }\end{array}$ \\
\hline Light pulse duration & $\sim 10 \mathrm{~ns}$ & $\sim 10 \mathrm{~ns}$ \\
\hline Configuration & Biaxial & Coaxial \\
\hline Resolution & $6 \mathrm{~m}$ & $6 \mathrm{~m}$ \\
\hline Scanning capability & Azimuth $0^{\circ}-180^{\circ}$, elevation $0^{\circ}-90^{\circ}$ & Azimuth $0^{\circ}-180^{\circ}$, elevation $0^{\circ}-90^{\circ}$ \\
\hline Max range & $12288 \mathrm{~m}$ (2048 bins) & $12288 \mathrm{~m}$ (2048 bins) \\
\hline Digitizers & Dual 12 bit, $125 \mathrm{MHz}$ & Dual 12 bit, $100 \mathrm{MHz}$ \\
\hline Pulse frequency & $15 \mathrm{~Hz}$ & $15 \mathrm{~Hz}$ \\
\hline Max energy per pulse & $98 \mathrm{~mJ}(1064 \mathrm{~nm}), 45 \mathrm{~mJ}(355 \mathrm{~nm})$ & $335 \mathrm{~mJ}(1064 \mathrm{~nm}), 185 \mathrm{~mJ}(532 \mathrm{~nm}), 44 \mathrm{~mJ}(355 \mathrm{~nm})$ \\
\hline
\end{tabular}



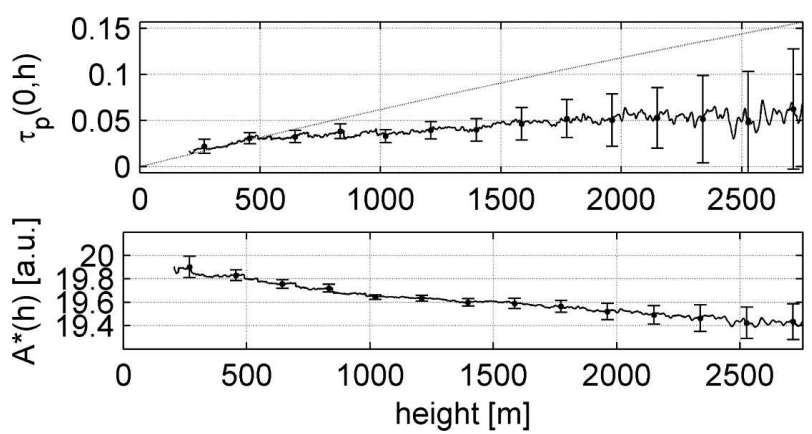

FIG. 6. FSL lidar data from the 355-nm channel, measured on 6 Apr 2005, sequence 1. (top) The particulate optical depth, $\tau_{p}(0, h)$, and the molecular optical depth, $\tau_{m}(0, h)$, shown as the thick and thin curves, respectively. (bottom) The intercept $A^{*}(h)$. The STD is shown by error bars.

stratified atmosphere) is better met for shorter wavelengths. Particularly, the measurement data at $355 \mathrm{~nm}$ obey qualitative requirements (i) and (ii) (see previous section) much better than the data at $1064 \mathrm{~nm}$. Therefore, we will focus on the inversion results obtained from the lidar signals at $355 \mathrm{~nm}$. A typical example of experimental data measured with the FSL lidar on 6 April 2005 (sequence 1) is shown in Fig. 6. The upper plot represents the particulate optical depth $\tau_{p}(0, h)$ and the molecular optical depth (the thick and thin curves, respectively), while the lower plot shows the intercept $A^{*}(h)$. Observe that $h_{\max }$ is $\sim 2700 \mathrm{~m}$, following the three criteria. Note, that in general, the STD for $\tau(0, h)$ is larger than the STD for $A^{*}(h)$. It follows from our theoretical and experimental results that the relative uncertainty of the profile of the intercept $A^{*}(h)$ is normally less than that for the optical depth; accordingly, this function can generally be used for analyses over a more extended height interval.

In Fig. 7, examples of (a) the optical depths and (b)
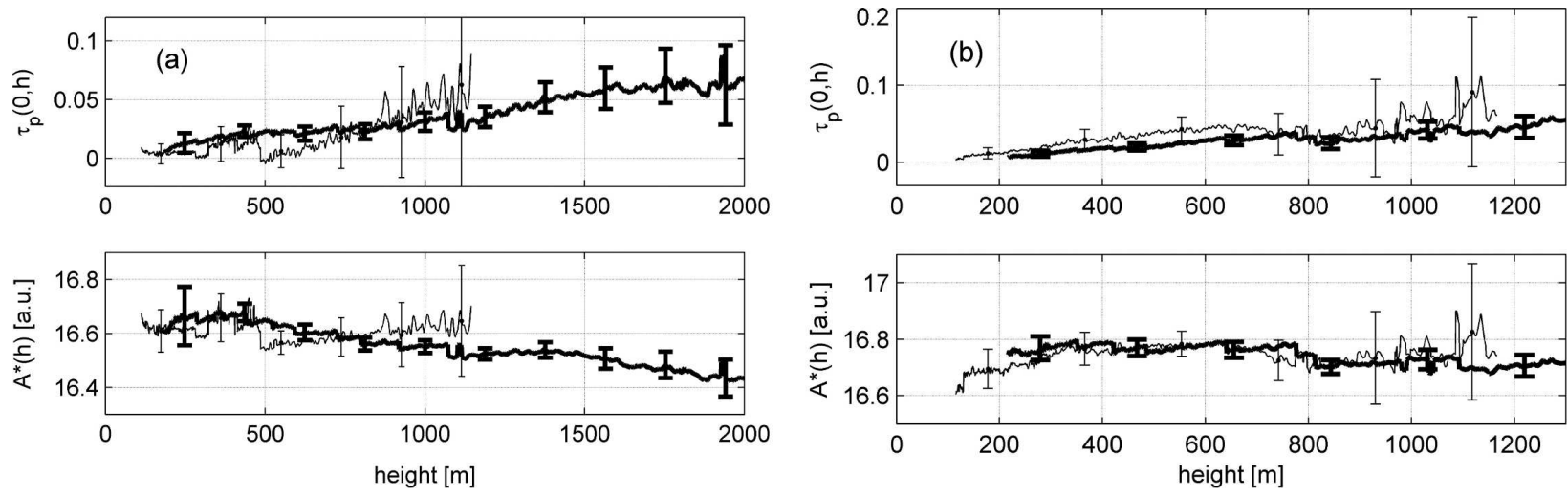

FIG. 7. (a) Particulate optical depth, $\tau_{p}(0, h)$, and the intercepts, $A^{*}(h)$, obtained with the two lidars [FSL (thick curves) and JHU (thin curves)] on 5 Apr 2005, sequence 1. Their STDs are shown by error bars. The FSL intercept functions are normalized to have the same scale as the JHU profiles. (b) Same as in (a), but obtained on 5 Apr 2005, sequence 2. 

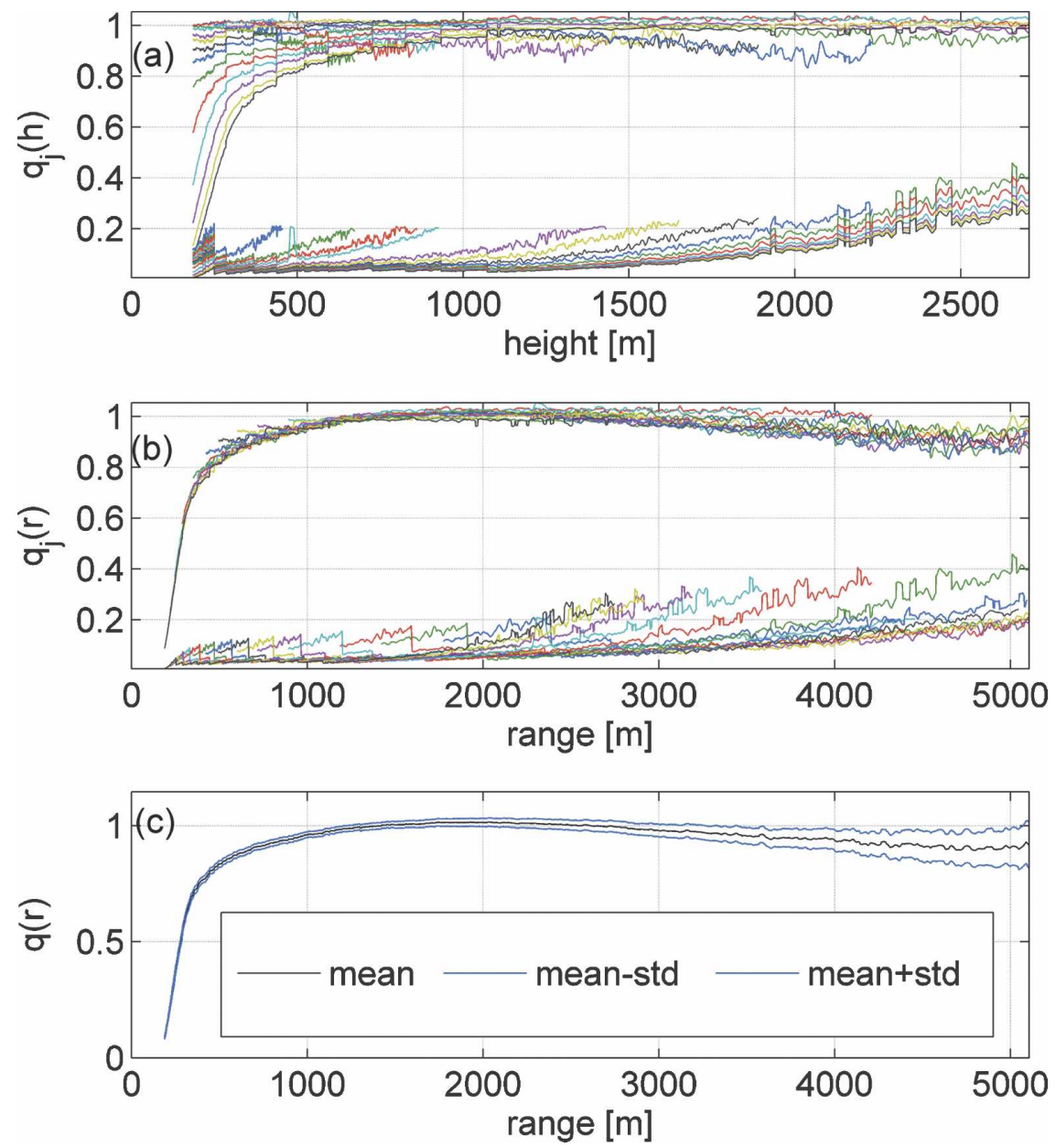

FIG. 8. Overlap retrieval. (a) Individual overlap function as a function of height $q_{j}(h)$; the lower curves represent the STD. (b) Individual overlap function as a function of range $q_{j}(r)$; the lower curves represent the STD. (c) Mean overlap (black curve) and its STD (blue curves).

tions allow one to calculate a vertical "synthetic" rangesquared-corrected signal, $Z_{90}^{*}(h)$,

$$
Z_{90}^{*}(h)=\exp \left[A^{*}(h)\right] \exp [-2 \tau(0, h)]
$$

and the corresponding synthetic signals along the elevation angles $\varphi_{j}$,

$$
Z_{j}^{*}(h)=\exp \left[A^{*}(h)\right] \exp \left[\frac{-2 \tau(0, h)}{\sin \varphi_{j}}\right]
$$

Using both synthetic and real signals for each elevation angle $\varphi_{j}$ [Eqs. (8) and (1), respectively], we are now in a position to calculate the lidar overlap function versus height along this direction as

$$
q_{j}(h)=\frac{P_{j}(h)\left(h / \sin \varphi_{j}\right)^{2}}{Z_{j}^{*}(h)} .
$$

The height-dependent functions $q_{j}(h)$, determined for different $\varphi_{j}$, are then recalculated as functions of the slope range, $q_{j}(r)$, and averaged to obtain the mean overlap function $q(r)$ (see Fig. 8). Hence, an estimate of the overlap function $q(r)$ can be determined directly from the multiangle measurement data, provided that the retrieved profiles $A^{*}(h)$ and $\tau(0, h)$ are free of systematic distortions. By comparing the averaged overlap function with the individual functions $q_{j}(r)$, one can also determine whether the retrieved individual overlap functions are affected by systematic distortions when the lidar elevation angles are changed during the scanning. Because of the high sensitivity of multiangle mea- 

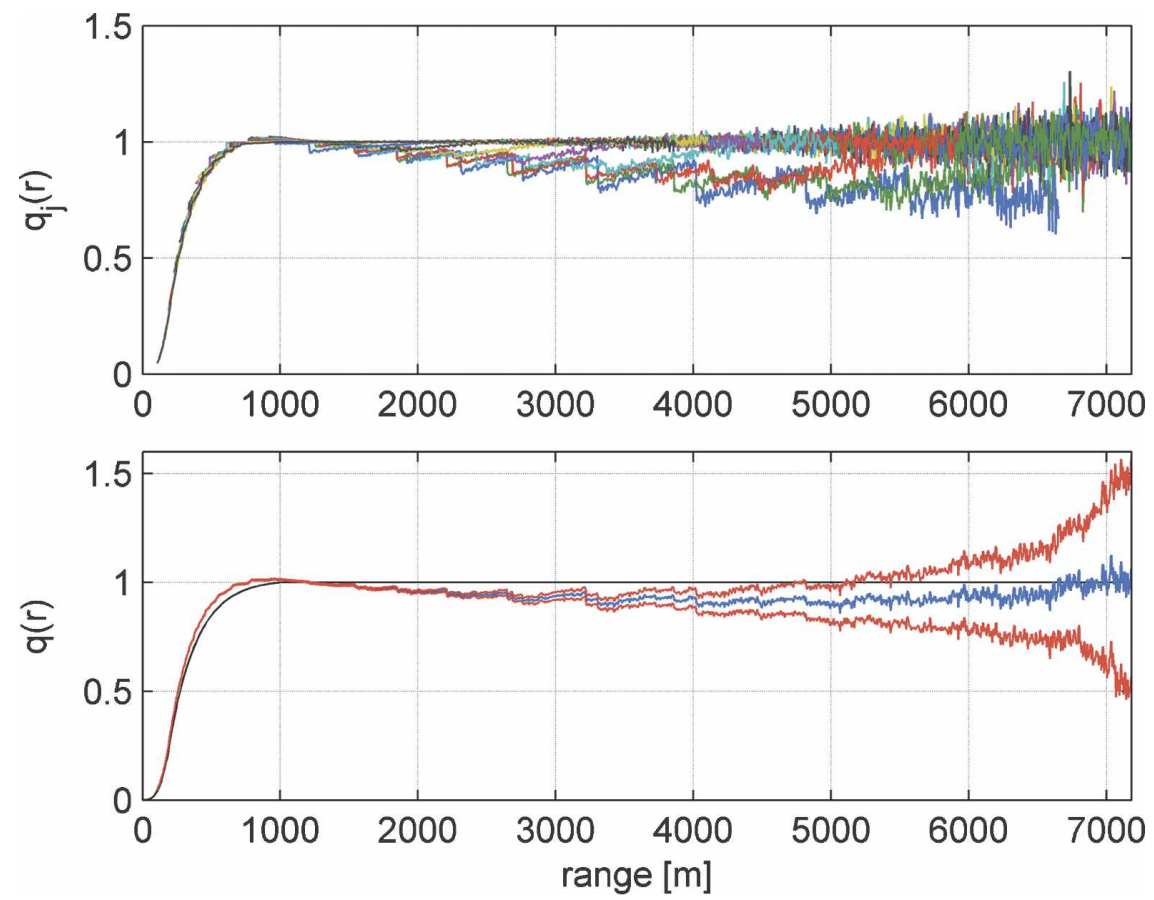

FIG. 9. (top) The retrieved $q_{j}(r)$ and (bottom) the mean function $q(r)$ and its STD (blue and red curves, respectively) obtained with an erroneously estimated length of the incompleteoverlap zone when determining $\tau_{p}(0, h)$ and $A^{*}(h)$ (recall Fig. 1$)$. The simulated lidar signals are corrupted by random noise ( $\mathrm{STD}=1$ count), the offset being zero. The overlap model is shown on the lower plot by the black thin curve. The STD is calculated using the weighted method (see the appendix).

surements to any systematic distortions, we believe that this method is a useful alternative to traditional methods of determining the overlap function (e.g., methods by Sasano et al. 1979; Sassen and Dodd 1982; Tomine et al. 1989; Dho et al. 1997).

Results of our numerical experiments showed that generally the presence of random signal noise does not significantly influence the mean overlap function. The influence of systematic signal distortions is usually more significant than noise. When data points from the incomplete-overlap zone are erroneously used to determine the slope and intersect of the linear regression, there will also be a slight increase of the value of the overlap function in the region of the incomplete overlap, followed by a noticeable decrease of the value of the overlap function in the intermediate range (here, 2000-4000 m). We illustrate this with an example, shown in Fig. 9. The individual values of $q_{j}(r)$ are shown in the upper plot where the deviation from unity for ranges of more than $1000 \mathrm{~m}$ is noticeable for an overlap corresponding to small elevation angles. The mean overlap function $q(r)$ (blue curve) and its STD (red curves) are shown on the lower plot. The incompleteoverlap zone is erroneously assumed to be $500 \mathrm{~m}$ (the actual length being $1000 \mathrm{~m}$ ). The correct overlap function is shown by the thin black curve on the lower plot. The simulated lidar signals are corrupted only by random noise (STD = 1 count), the offset being zero. The maximum signals reach 3100-3300 counts. Recall Fig. 1 and note how the underestimated inversion results $\tau_{p}(0$, $h)$ and $A^{*}(h)$ are reflected by the overlap function. Figures 10 and 11 show the retrieved overlap function for the case of systematic distortion due to offset in all signals (while the region of the incomplete overlap is estimated correctly). The background component is underestimated and overestimated by 1 count (such that the offset $= \pm 1$ count), respectively, and the signals are also corrupted by random noise $(\mathrm{STD}=1$ count). All three criteria (described in the methodology section) are applied for the inversion. The observed effects on $q(r)$ for the two cases (offset $= \pm 1$ count) are opposite, but the effect due to the negative offset -1 (background component overestimated) is noticeably strong in the far field. A comparison with Fig. 2 reveals that the estimates of $\tau(0, h)$ and $A^{*}(h)$ are reflected in the overlap profile. An overestimated $\tau(0, h)$ and $A^{*}(h)$ will result in an underestimated overlap for far ranges (for more than $3000 \mathrm{~m}$ in this particular case) and vice 

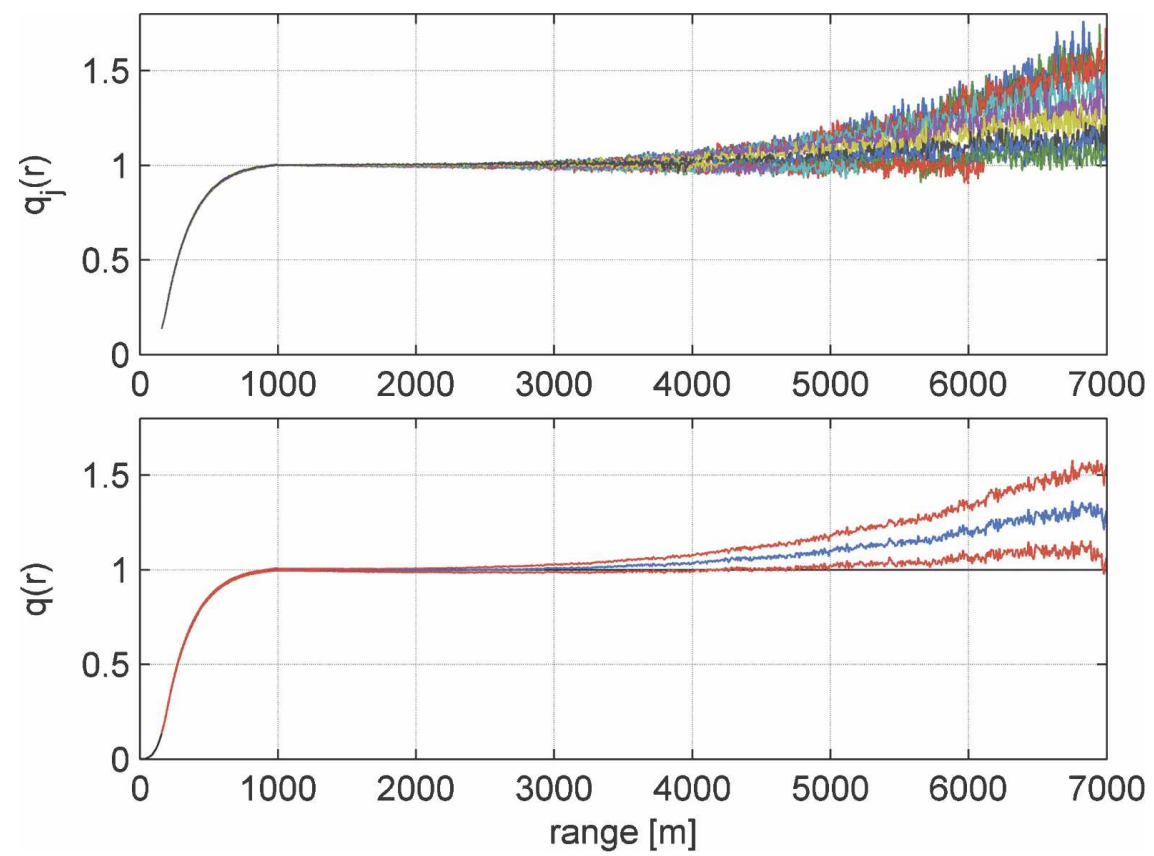

FIG. 10. (top) The retrieved $q_{j}(r)$ and (bottom) the mean function $q(r)$ and its STD (blue and red curves, respectively) obtained when all signals are corrupted by a systematic offset of (top) +1 count. (bottom) The mean overlap $q(r)$ (blue curve) and its STD (red curves). The overlap model is shown by the thin black curve on the lower plot. The length of the incomplete overlap is correctly assumed $(1000 \mathrm{~m})$.

versa. For experimental data the shape of the overlap function provides additional information regarding the accuracy of the retrieved $\tau(0, h)$ and $A *(h)$. It should be noted that, because multiangle methods are extremely sensitive to any instrumental distortions in lidar data, multiangle methods to determine the overlap function may be useful when performing lidar tests and calibrations.
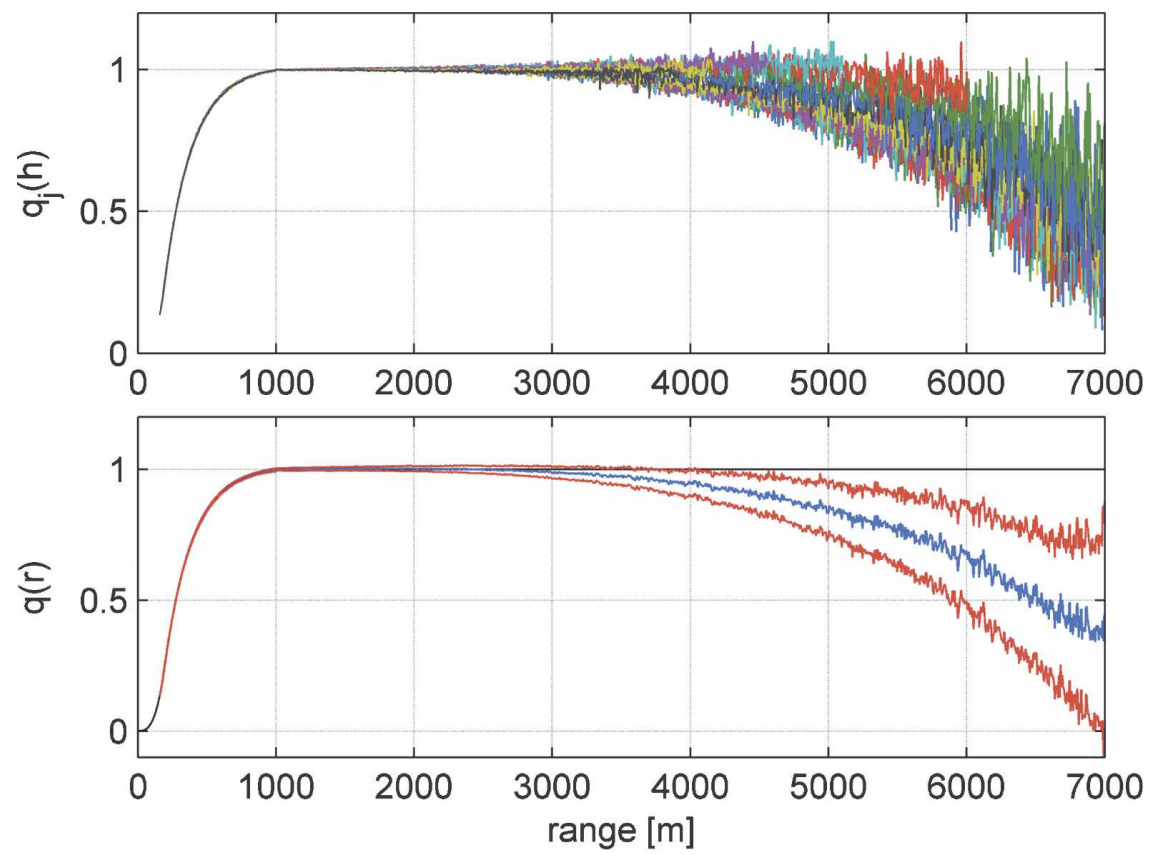

FIG. 11. Same as in Fig. 7, but here the functions $q_{j}(r)$ are obtained when all signals are corrupted by a systematic offset of -1 count. 

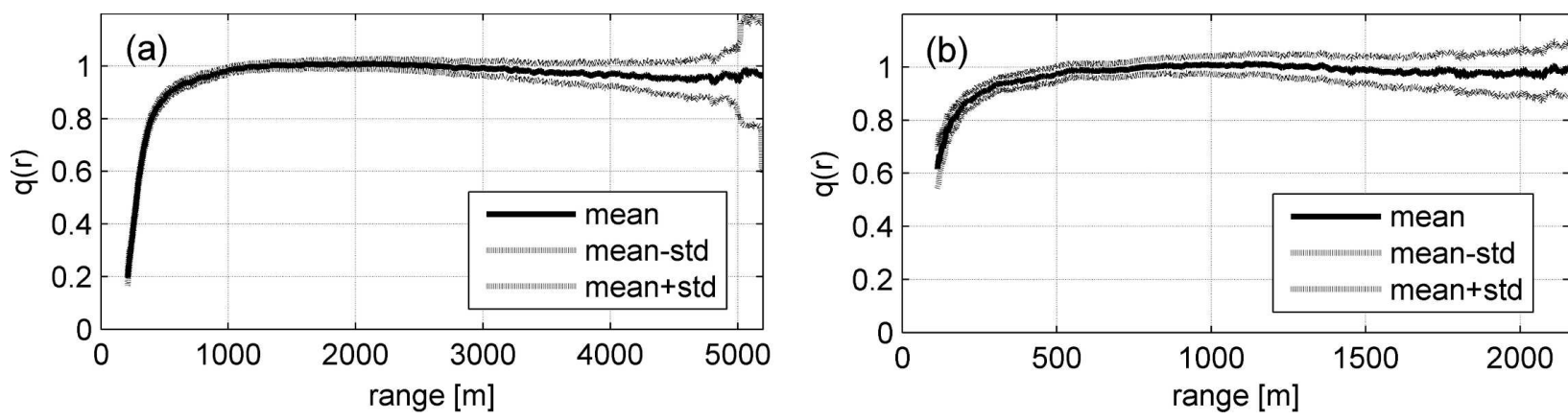

FIG. 12. The overlap functions for the 355-nm channel for (a) the FSL lidar and (b) the JHU lidar. The dotted curves represent the STD boundaries.

\section{b. Experiment}

The overlap functions for the 355-nm channel of the FSL and JHU lidars are shown in Figs. 12a and 12b, respectively. These plots represent mean overlap profiles and their STDs, computed using a weighted method (with the weights given by the individual overlaps variance; see the appendix). In favorable conditions (cloud free), which occurred during the last days of the experiment, the FSL lidar overlap could be accurately determined up to $5000 \mathrm{~m}$, while the JHU overlap could be determined up to $\sim 2000 \mathrm{~m}$. For the FSL lidar, the complete-overlap zone starts at $\sim 1200 \mathrm{~m}$, and the JHU lidar complete-overlap zone starts at $\sim 600 \mathrm{~m}$. The behavior of the retrieved overlap function $q(r)$ in the more distant zones of the complete overlap, particularly its deflection from unity, is strongly related to the quality of measured data, that is, on the presence or absence of offsets in the signals.

Last, consider the consequences of an incorrect background subtraction on the behavior of the retrieved overlap function, illustrated in Fig. 13. Here the overlap functions found for the last two elevation angles $\left(68^{\circ}\right.$ and $\left.80^{\circ}\right)$ of the measurement sequence are underestimated because of an overestimated background. However, in this particular case, the mean overlap is only slightly affected by the two particular angles in this case. Hence, we can infer that the $\tau_{p}(0, h)$ and $A^{*}(h)$ are also slightly overestimated.

The systematic distortions that corrupt the overlap function must be removed (or at least minimized). The flowchart of determining the optical depth and intercept, on the one hand, and the overlap, on the other

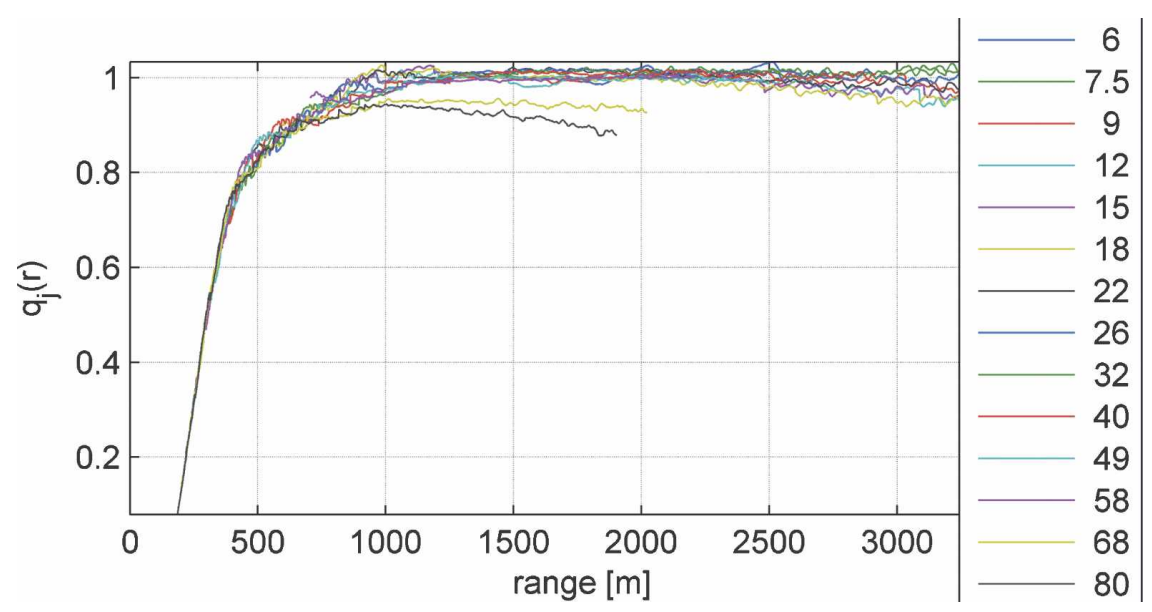

FIG. 13. Example of the overlap functions obtained from data with an inaccurate background subtraction. The overlap functions are obtained from the FSL 355-nm data on 6 Apr 2005, sequence 2 . The plot shows the overlaps, $q_{j}(r)$, for all elevation angles; one can clearly see the underestimated overlaps obtained for the data measured in the slope directions $68^{\circ}$ and $80^{\circ}$. 


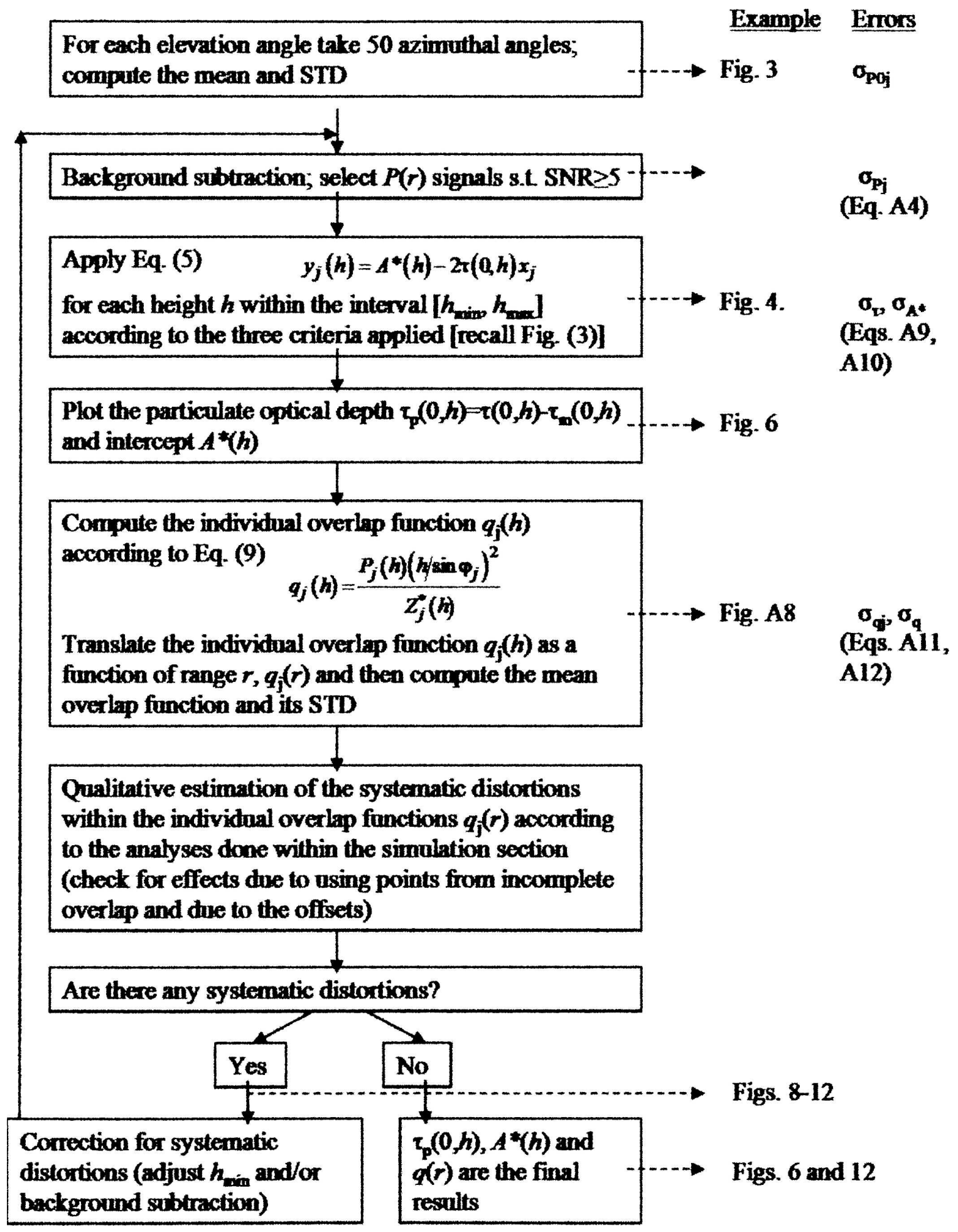

FIG. 14. Flowchart for the measurement technology and data-processing technique.

hand, is shown in Fig. 14. The experimental results shown in the present paper (optical depth and intercept, as well as the overlap) are obtained after iterating according to the flowchart. The individual overlap func- tions show us if there are systematic distortions in the lidar signals. This allows us to correct for $h_{\min }$ and background subtraction and improve the accuracy of the optical depth and intercept. More detailed analysis of 
the systematic distortions is presented in the study by Kovalev et al. (2007, manuscript submitted to Appl. Opt.).

\section{Summary}

The purpose of this study is to analyze and improve the Kano-Hamilton method for inversion of multiangle data, obtained with an elastic lidar under clear atmospheric conditions. It is generally agreed that the inaccuracy of multiangle measurements results from applying a method that assumes homogeneity in situations where the atmosphere is heterogeneous. Our analysis revealed that the problem is in fact more cumbersome. We showed that even minor errors that stem from instrumental uncertainties, inherent to real lidar data, have important consequences for the Kano-Hamilton method. This could significantly impede the use of this method even in an ideally homogeneous atmosphere. We conclude that the method needs to be improved in order to be applied to real experimental data. In the current paper we propose ways of improving the measurement methodology when determining and analyzing both constants in Eq. (5) and the behavior of the overlap functions, retrieved from the functions $A *(h)$ and $\tau(0, h)$. The basic principle of our approach is to thoroughly identify those data points that distort the linear dependence of the function $y_{j}(h)$ on $x_{j}$ and remove them. A quantitative analysis, considering error propagation, was performed including the STD of the raw mean signals $P(r)$ as input source for the errors. Additionally, we analyzed the uncertainty in the retrieved data, taking into consideration both random signal errors (see the appendix) and systematic distortions (Fig. 14). Our current study also includes a new principle for the determination of effective overlap for scanning lidar that operates in clear atmospheres. With this procedure the actual behavior of the overlap function in the incomplete-overlap zone can be determined, and useful information on the quality of derived atmospheric parameters can be obtained. In particular, this procedure allows a determination of whether a significant offset remains in the inverted data after background subtraction and whether the atmospheric conditions under consideration are suitable for multiangle measurements with the Kano-Hamilton approach.

The application of the modified Kano-Hamilton method to experimental data obtained in clear atmospheres at different wavelengths showed that the lidar signals measured at $355 \mathrm{~nm}$ yield inversion results with higher accuracy than signals measured at longer wavelengths. Our results show that the retrieved profiles of $\tau_{p}(0, h)$ at $355 \mathrm{~nm}$ better meet conditions (i) and (ii) (see section on methodology) than the signals at longer wavelengths. In most situations and for the clear atmospheric conditions investigated here, the function $A^{*}(h)$ and, accordingly, the total backscatter coefficient, monotonically decrease with height.

The application of the multiangle method to the data from the 532- and 1064-nm channels yielded less encouraging results. The total optical depth for these wavelengths over the same altitude range is less than that for $355 \mathrm{~nm}$, and the signals are much more sensitive to the heterogeneity of particulate loading. In addition, the backscatter signals at $1064 \mathrm{~nm}$ are much weaker than the signals at $355 \mathrm{~nm}$, the signal-to-noise ratio is worse and, accordingly, the total measurement range at this wavelength is much shorter than at $355 \mathrm{~nm}$. The lidar measurements at $532 \mathrm{~nm}$ are more promising, and we suspect that this wavelength can potentially be used successfully in multiangle measurements in clear atmospheres.

The selection of an optimal measurement range $\left(h_{\min }, h_{\max }\right)$ of the lidar signals for the inversion is another important consideration when making multiangle measurements. To accurately select the minimum range, $h_{\min }$, the length of the incomplete-overlap zone must be accurately determined, and systematic signal distortions must not be present in areas of sharp changes in the signal intensities. The selection of the maximum range, $h_{\max }$, for the inversion is as important as the selection of the minimal range, $h_{\min }$. The random signal noise at the far end of the measurement range, an effect that strongly increases with range, coupled with systematic distortions due to a remaining offset, may result in large systematic shifts in the linear regression of the dependence of $y_{j}(h)$ on $x_{j}$. In the present work, $h_{\max }$ was restricted by the condition SNR $>5$ and by criterion 3 (use of a sufficient number of points for regression at $h_{\max }$, here at least 6). Through combining these considerations to reach the optimal height interval used for inversion, we have developed a general technique that will improve the analysis of multiangle lidar measurements. Further work includes the determination of the aerosol extinction coefficient, using an iterative method, taking into account both the optical depth and the relative backscatter coefficient (Kovalev et al. 2006; Kovalev 2006).

\section{APPENDIX}

\section{Error Propagation}

\section{a. Background}

Recall the error propagation for two general cases. Assume that $y$ is a function of $x_{i}$; that is, $y\left(x_{1}, x_{2}, \ldots\right)=$ $\Sigma_{i} x_{i}$. 
The error in $y$ is determined as

$$
\sigma_{y}=\sqrt{\sum_{i}\left(\frac{\partial y}{\partial x_{i}} \sigma_{x_{i}}\right)^{2}}=\sqrt{\sum_{i} \sigma_{x_{i}}^{2}}
$$

Now, assume $y$ is a function of $x_{i}$ and $z_{j}$; that is, $y\left(x_{1}\right.$, $\left.x_{2}, \ldots, z_{1}, z_{2}, \ldots\right)=\left(\Pi_{i} x_{i} / \Pi_{j} z_{j}\right)$. The error in $y$ will be

$$
\begin{aligned}
\sigma_{y} & =\sqrt{\sum_{i}\left(\frac{\partial y}{\partial x_{i}} \sigma_{x_{i}}\right)^{2}+\sum_{j}\left(\frac{\partial y}{\partial z_{j}} \sigma_{z_{j}}\right)^{2}} \\
& =\sqrt{\sum_{i}\left(\frac{y}{x_{i}} \sigma_{x_{i}}\right)^{2}+\sum_{j}\left(-\frac{y}{z_{j}} \sigma_{z_{j}}\right)^{2}} \\
& =y \sqrt{\sum_{i}\left(\frac{\sigma_{x_{i}}}{x_{i}}\right)^{2}+\sum_{j}\left(\frac{\sigma_{z_{j}}}{z_{j}}\right)^{2}} .
\end{aligned}
$$

b. Mean and STD for the raw signals

As mentioned, the mean signal $P_{0 j}(r)$, where $j$ specifies the elevation angle $\varphi_{j}$, is determined as the mean of the number of horizontal scans. Its STD, $\sigma_{P 0 j(r)}$, accounts for the error propagation within the calculations. After the background subtraction, the signal and its STD, as a function of range, are

$$
\begin{aligned}
P_{j}(r) & =P_{0 j}(r)-B_{j}(r), \\
\sigma_{P_{j}(r)} & =\sqrt{\sigma_{P_{0 j}(r)}^{2}+\sigma_{B_{j}(r)}^{2}} .
\end{aligned}
$$

$\mathrm{SNR}$ is defined as the ratio $P_{j}(r) / \sigma_{P j(r)}$.

\section{c. Mean and STD for intercept and optical depth}

The intercept and optical depth ( -0.5 slope) in Eq. (5) are computed using a weighted method (see Taylor 1997), where the weights $w_{j}(h)$ are given by the STD of $y_{j}(h)$ :

$$
w_{j}(h)=\sigma_{y_{j}(h)}=\sigma_{P_{j}(h)} / P_{j}(h) .
$$

Note that the mean and STD are given now as a function of height:

$$
\begin{aligned}
\tau(0, h) & =-\frac{1}{2} \frac{1}{\Delta}\left[\sum \frac{1}{w_{j}^{2}(h)} \sum \frac{1}{w_{j}^{2}(h)} x_{j} y_{j}(h)-\sum \frac{1}{w_{j}^{2}(h)} x_{j} \sum \frac{1}{w_{j}^{2}(h)} y_{j}(h)\right], \\
A^{*}(h) & =\frac{1}{\Delta}\left[\sum \frac{1}{w_{j}^{2}(h)} x_{j}^{2} \sum \frac{1}{w_{j}^{2}(h)} y_{j}(h)-\sum \frac{1}{w_{j}^{2}(h)} x_{j} \sum \frac{1}{w_{j}^{2}(h)} x_{j} y_{j}(h)\right],
\end{aligned}
$$

where $\Delta$ is

$$
\Delta=\sum \frac{1}{w_{j}^{2}(h)} \sum \frac{1}{w_{j}^{2}(h)} x_{j}^{2}-\left[\sum \frac{1}{w_{j}^{2}(h)} x_{j}\right]^{2} .
$$

The STD for intercept and optical depth are

$$
\begin{gathered}
\sigma_{\tau}=\sqrt{\frac{\sum \frac{1}{w_{j}^{2}(h)} x_{j}^{2}}{\Delta}}, \\
\sigma_{A^{*}}=\sqrt{\frac{\sum \frac{1}{w_{j}^{2}(h)}}{\Delta}} .
\end{gathered}
$$

\section{d. Mean and STD for the overlap}

The STD of the individual overlap [Eq. (9)], according to the propagation of the errors, is

$$
\sigma_{q_{j}(h)}=q_{j}(h) \sqrt{\left[\frac{\sigma_{P_{j}(h)}}{P_{j}(h)}\right]^{2}+\sigma_{A^{*}(h)}^{2}+4 x_{j}^{2} \sigma_{\tau(0, h)}^{2}} .
$$

The mean overlap is determined using a weighting method of the individual overlaps $q_{j}(r)$ :

$$
q(r)=\frac{\sum \frac{q_{j}(r)}{\sigma_{q_{j}(r)}^{2}} .}{\sum \frac{1}{\sigma_{q_{j}(r)}^{2}} .}
$$

The corresponding STD is

$$
\sigma_{q(r)}=\frac{1}{\sqrt{\sum \frac{1}{\sigma_{q_{j}(r)}^{2}}}} .
$$

\section{REFERENCES}

Barlow, R. J., 1989: Statistics: A Guide to the Use of Statistical Methods in the Physical Sciences. Wiley, 204 pp.

Böckmann, C., and Coauthors, 2004: Aerosol lidar intercomparison in the framework of the EARLINET project. 2. Aerosol backscatter algorithms. Appl. Opt., 43, 977-989.

Bristow, M., 2002: Suppression of afterpulsing in photomultipliers by gating the photocathode. Appl. Opt., 41, 4975-4987.

Dho, S. W., Y. J. Park, and H. J. Kong, 1997: Experimental determination of a geometric form factor in a lidar equation for an inhomogeneous atmosphere. Appl. Opt., 36, 6009-6010. 
Fang, H.-T., and D.-S. Huang, 2004: Noise reduction in lidar signal based on discrete wavelet transform. Opt. Commun., 233, $67-76$.

Hamilton, P. M., 1969: Lidar measurement of backscatter and attenuation of atmospheric aerosol. Atmos. Environ., 3, 221223.

Kano, M., 1968: On the determination of backscattered and extinction coefficient of the atmosphere by using laser radar. Pap. Meteor. Geophys., 19, 121-129.

Kovalev, V. A., 2002: Analytical differentiation of the differentialabsorption-lidar data distorted by noise. Appl. Opt., 41, 11561162.

— 2004: Distortions of the extinction coefficient profile caused by systematic errors in lidar data. Appl. Opt., 43, 3191-3198.

_ 2 2006: Determination of slope in lidar data using a duplicate of the inverted function. Appl. Opt., 45, 8781-8789.

-, and W. E. Eichinger, 2004: Elastic Lidar: Theory, Practice, and Analysis Methods. Wiley, $615 \mathrm{pp}$.

— C. Wold, J. Newton, and W. M. Hao, 2004: Combination of one-directional and multiangle data processing techniques to reduce uncertainty in the elastic lidar measurement data. Proc. SPIE Laser Radar Techniques for Atmospheric Sensing, Vol. 5575, Gran Canarias, Spain, SPIE, 82-90.

,,$-- \ldots$, and 2006: Determination of extinction coefficient profiles from multiangle lidar data using a "clone" of the optical depth. Proc. 23rd Int. Laser Radar Conf. on Lidar Remote Sensing in Atmospheric and Earth Sciences, Nara, Japan, INDECO Inc., 283-286.

— W. M. Hao, C. Wold, and M. Adam, 2007: Experimental method for the examination of systematic distortions in lidar data. Appl. Opt., 46, 6710-6718.

Kunz, G. J., and G. de Leeuw, 1993: Inversion of lidar signals with the slope method. Appl. Opt., 32, 3249-3256.

Lee, H. S., G. K. Schwemmer, C. L. Korb, M. Dombrowski, and C. Prasad, 1990: Gated photomultiplier response characterization for DIAL measurements. Appl. Opt., 29, 3303-3315.

Pahlow, M., V. A. Kovalev, and M. B. Parlange, 2004: Calibration method for multiangle lidar measurements. Appl. Opt., 43, 2948-2956.

Rocadenbosch, F., A. Comeron, and D. Pineda, 1998: Assessment of lidar inversion errors for homogeneous atmospheres. Appl. Opt., 37, 2199-2206.

, - — and L. Albiol, 2000: Statistics of the slope-method estimator. Appl. Opt., 39, 6049-6057.

—, M. Sicard, and A. Comeron, 2004: Automated variableresolution algorithm for Raman extinction retrieval. Proc. 22nd Int. Laser Radar Conf. on Lidar Remote Sensing in
Atmospheric and Earth Sciences, Matera, Italy, European Space Agency, 467-470.

Rothermel, J., and W. Jones, 1985: Ground-based measurements of atmospheric backscatter and absorption using coherent $\mathrm{CO}_{2}$ lidar. Appl. Opt., 24, 3487-3496.

Russell, P. B., and J. M. Livingston, 1984: Slant-lidar aerosol extinction measurements and their relation to measured and calculated albedo changes. J. Appl. Meteor., 23, 1204-1221.

Sasano, Y., 1996: Tropospheric aerosol extinction coefficient properties derived from scanning lidar measurements over Tsukiba, Japan, from 1990 to 1993. Appl. Opt., 35, 4941-4952.

— - H. Shimizu, N. Takeuchi, and M. Okuda, 1979: Geometrical form factor in the laser radar equation: An experimental determination. Appl. Opt., 18, 3908-3910.

Sassen, K., and G. C. Dodd, 1982: Lidar crossover function and misalignment effects. Appl. Opt., 21, 3162-3165.

Sicard, M., P. Chazette, J. Pelon, J. G. Won, and S. C. Yoon, 2002: Variational method for the retrieval of the optical thickness and the backscatter coefficient from multiangle lidar profiles. Appl. Opt., 41, 493-502.

Spinhirne, J. D., J. A. Reagan, and B. M. Herman, 1980: Vertical distribution of aerosol extinction cross section and inference of aerosol imaginary index in the troposphere by lidar technique. J. Appl. Meteor., 19, 426-438.

Takamura, T., Y. Sasano, and T. Hayasaka, 1994: Tropospheric aerosol optical properties derived from lidar, sun photometer, and optical particle counter measurements. Appl. Opt., 33, 7132-7140.

Taylor, J. R., 1997: An Introduction to Error Analysis: The Study of Uncertainties in Physical Measurements. University Science Books, 327 pp.

Tomine, K., C. Hirayama, K. Michimoto, and N. Takeuchi, 1989: Experimental determination of the crossover function in the laser radar equation for days with a light mist. Appl. Opt., 28, 2194-2195.

Utkin, A. B., A. V. Lavrov, L. Costa, F. Simões, and R. Vilar, 2002: Detection of small forest fires by lidar. Appl. Phys., 74, 77-83.

Volkov, S. N., B. V. Kaul, and D. I. Shelefontuk, 2002: Optimal method of linear regression in laser remote sensing. Appl. Opt., 41, 5078-5083.

Whiteman, D. N., 1999: Application of statistical methods to the determination of slope in lidar data. Appl. Opt., 38, 33603369.

Zhao, Y., 1999: Signal-induced fluorescence in photomultipliers in differential absorption lidar systems. Appl. Opt., 38, 46394648 . 